\title{
Infectious Risks and Optimal Strength of Maintenance Immunosuppressants in Rituximab-Treated Kidney Transplantation
}

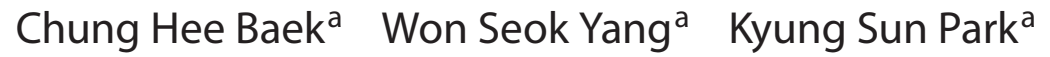 \\ Duck Jong Han ${ }^{\mathrm{b}}$ Jae Berm Park ${ }^{\mathrm{b}}$ Su-Kil Park ${ }^{\mathrm{a}}$ \\ a Division of Nephrology, Department of Internal Medicine, and bepartment of Surgery, \\ Asan Medical Center, University of Ulsan College of Medicine, Seoul, Republic of Korea
}

\section{Key Words}

Kidney transplantation $\cdot$ Mycophenolate mofetil $\cdot$ Rituximab

\begin{abstract}
Background: Rituximab, an anti-CD20 antibody, effectively depletes B lymphocytes. It is not clear whether the use of conventional doses of mycophenolate mofetil (MMF), methylprednisolone and tacrolimus as maintenance immunosuppression in rituximab-treated kidney transplantation is associated with increased risk. Methods: We retrospectively evaluated 67 patients who underwent $\mathrm{HLA}$-sensitized or ABO-incompatible living donor kidney transplantation after one dose of rituximab (200 or 500 mg) (group 1). Eighty-seven kidney transplant recipients who did not require rituximab served as a control (group 2). Results: Cytomegalovirus infection (16.4 vs. $5.7 \%, p=0.031$ ) and pneumonia ( 9.0 vs. $1.1 \%, p=0.043$ ) occurred more often in group 1 , and 2 patients of group 1 died of infection. The doses of methylprednisolone and tacrolimus levels of the two groups were not different. MMF dose was reduced when serious infection occurred. The doses of MMF (in grams/day) at the following times postoperatively were lower in group 1 than in group 2: 1 month: $1.26 \pm 0.42$ vs. $1.40 \pm 0.39, p=0.033 ; 3$ months: $1.14 \pm 0.51$ vs. 1.36 $\pm 0.39, \mathrm{p}=0.011 ; 6$ months: $1.07 \pm 0.50$ vs. $1.30 \pm 0.42, \mathrm{p}=0.012 ; 1$ year: $0.88 \pm 0.52$ vs. 1.19 $\pm 0.44, p=0.009 ; 2$ years: $0.69 \pm 0.55$ vs. $1.25 \pm 0.49, p=0.059$, but the reduction of MMF doses did not increase the incidence of acute rejection in group 1 (4.5\% in group 1 vs. $9.2 \%$ in group $2, p=0.351$ ). If patients who died with functioning graft were excluded, graft survival was $98.5 \%$ in group 1 and $100 \%$ in group 2. Conclusions: Serious infectious complications were increased in rituximab-treated kidney transplant recipients and it might be adequate to reduce the MMF dose from the early postoperative period.

Copyright $\odot 2012$ S. Karger AG, Basel
\end{abstract}




\section{Introduction}

ABO-incompatible kidney transplantation (ABO-IKT) was started to increase the donor pool. In the 1980s, Alexandre et al. [1] performed ABO-IKT using plasmapheresis for removal of isohemagglutinins prior to transplantation, and hyperacute rejection was prevented. Since 1989, more than 1,000 ABO-IKT have been performed in Japan [2]. In the 2000s, rituximab, a chimeric monoclonal antibody against the protein CD20 which is primarily found on the surface of B lymphocytes, was introduced for the management of ABO-IKT [3, 4]. This protocol has become standard in multiple centers after Tydén et al. [5] first introduced antigen-specific immunoabsorption combined with anti-CD20 antibody and Sonnenday et al. [6] reported successful ABO-IKT using plasmapheresis, cytomegalovirus (CMV) hyperimmune globulin, and anti-CD20 antibody without splenectomy. Thereafter, the number of ABO-IKT with rituximab expanded, and long-term outcomes have been reported to be good $[7,8]$. Transplantations in patients with HLA sensitization have been performed relatively recently. In a recent report of 211 HLA-sensitized living donor kidney transplantations (KT), transplantation after desensitization provided a significant survival benefit compared with waiting for a compatible organ [9].

Potential complications from infection after splenectomy could be reduced if this is replaced by rituximab in ABO-IKT. However, infection is still a major cause of morbidity and mortality of renal transplant recipients. In a study of 1,218 renal transplant recipients, infection (29\%) was the second cause of death just after cardiovascular diseases (38\%) in these renal transplant recipients [10]. Recently, efforts to decrease the infectious complications by reducing immunosuppressant are ongoing [11-14]. However, it is not known whether standard doses of maintenance immunosuppressants are adequate in these patients who were preconditioned with rituximab.

In 2009, our center adopted a desensitization protocol for ABO-IKT or HLA-sensitized $\mathrm{KT}$, based on plasmapheresis, rituximab and standard immunosuppressive therapy. In this study, we retrospectively evaluated the risk of infectious complications in the incompatible $\mathrm{KT}$ preconditioned with rituximab, and assessed whether the standard maintenance immunosuppression could be reduced without increasing the risk of rejection.

\section{Patients and Methods}

\section{Patients}

Between January 2009 and May 2011, 80 patients with end-stage renal disease (ESRD) underwent $\mathrm{ABO}$-incompatible or HLA-sensitized living donor KT after preconditioning with rituximab, but without splenectomy, at Asan Medical Center in Seoul, Korea. The patients received tacrolimus, mycophenolate mofetil (MMF), and corticosteroids as maintenance immunosuppressants. Of the 80 patients, 13 patients used other immunosuppressants because of side effects or pregnancy. These patients were excluded and the remaining $67 \mathrm{pa}-$ tients were included in the analysis (group 1). As a control group, 87 living donor kidney transplant recipients who underwent compatible KT during the same period in our institution and received tacrolimus, MMF, and corticosteroids as maintenance immunosuppressants, but not rituximab (group 2) were compared with group 1 . This study was approved by our local institutional review board (2011-0426).

\section{Immunosuppression Protocol}

The immunosuppression protocol of rituximab-treated renal transplantation is summarized in figure 1. Immunosuppressants consisting of tacrolimus, MMF, and methylprednis- 
Fig. 1. Immunosuppressive protocol. Rituximab was administered and immunosuppressants (tacrolimus, MMF and mPD) were started 7-10 days before the operation. 1-6 sessions of plasmapheresis were performed prior to the operation, and basiliximab (anti-CD25 monoclonal antibody) was administered on the day of operation and postoperatively on day 4.

Fig. 2. Mortality. Three patients (4.5\%) died in group 1 whereas no mortality case was present in group $2(\mathrm{p}=0.053)$.
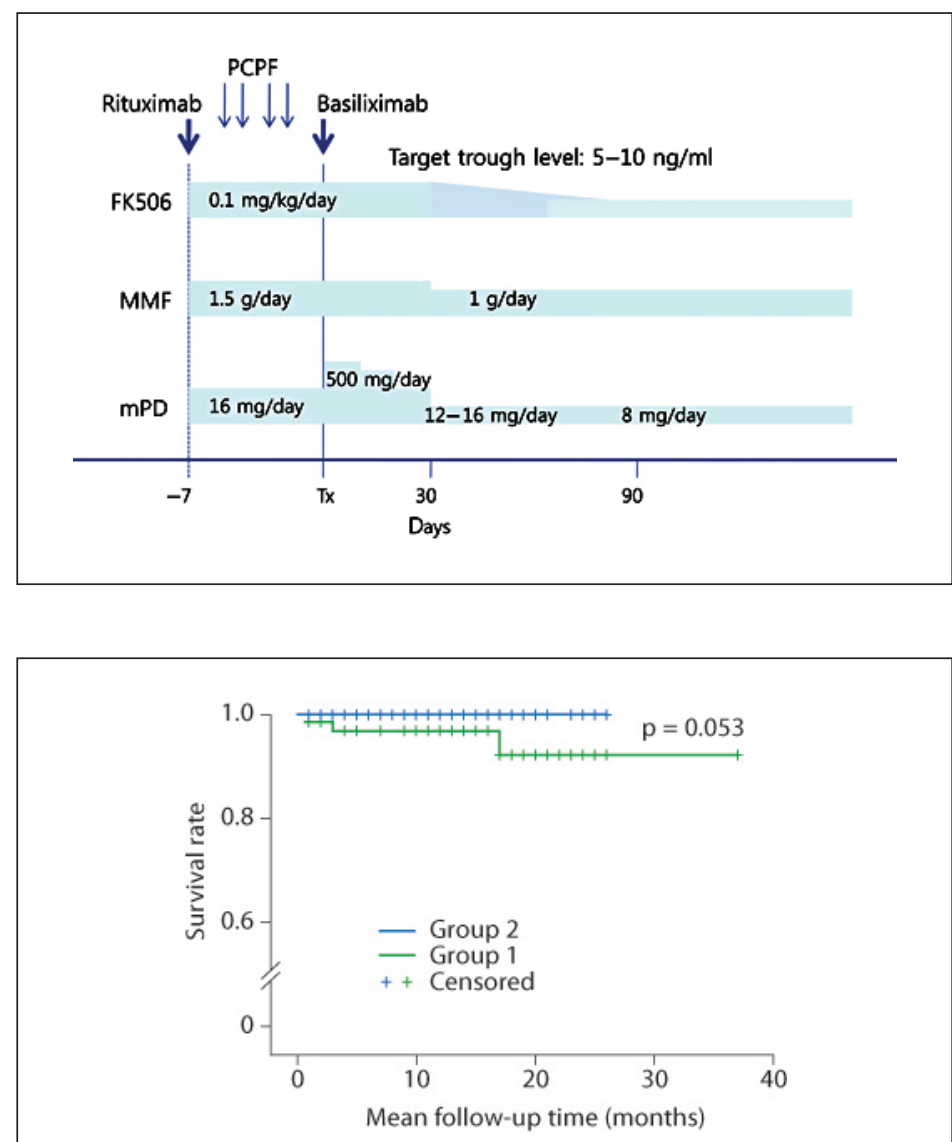

olone (mPD) were started 7-10 days before the operation. 1-6 sessions of plasmapheresis were performed 3-14 days prior to the operation until anti-A/B antibody titers decreased to a level below 1:4 or T flow cytometry was negative. Plasmapheresis removed immunoglobulin using post-centrifugal plasma filtration by blood cell separator (Cobe Spectra, Caridian BCT, USA) and filter (Evaflux 2A, Kawasumi Laboratories, Japan). Rituximab was administered 7-10 days before the operation. We used a single dose of $500 \mathrm{mg} /$ body until November 2009 and reduced the dose to $200 \mathrm{mg} /$ body since then. However, $500 \mathrm{mg} /$ body rituximab was administered in patients who were positive on crossmatching with flow cytometry. Thus, rituximab was administered as $200 \mathrm{mg}$ in 33 patients and $500 \mathrm{mg}$ in 47 patients. All the patients received an induction therapy with anti-CD25 monoclonal antibody (basiliximab) on the day of operation and postoperative day 4. Intravenous immunoglobulin (IVIG) was not administered.

In the control patients, anti-CD25 monoclonal antibody was administered on the day of operation and postoperative day 4 , which is the same as in rituximab-treated patients. Tacrolimus, MMF and $\mathrm{mPD}$ were started 2-3 days before the operation. IVIG was not administered.

\section{Detection of Anti-A/B Antibody Titers}

Evaluation of $\mathrm{ABO}$ isoagglutinin titers using tube test was applied to measure anti-A/B antibody titers. Anti-A/B isoagglutinin titers were determined by incubating $0.1 \mathrm{ml}$ of type A and B RBC suspensions in $0.1 \mathrm{ml}$ of saline containing 2-fold serial dilutions of patient's serum at room temperature. The agglutinating activity of IgM isoagglutinin was confirmed 
by treating the patient's serum with $0.01 \mathrm{mmol} / \mathrm{l}$ dithiothreitol, which abolished its agglutinating property.

\section{HLA Typing and Crossmatch}

HLA was typed with absolute HLA PCR/SSP kit (BioSewoom Inc., Korea) using sequence-specific primer. To exclude false negatives, control primer was used as positive control of PCR. HLA crossmatch was performed with T flow cytometry. Mean fluorescence intensity (MFI) $\geq 2.0$ was read as positive.

\section{Diagnosis of Rejection}

Surveillance biopsies were not performed routinely. When clinically indicated by rising serum creatinine or decreasing urine output, renal biopsies were performed to confirm rejection. C4d staining was performed with standard immunofluorescence techniques in all specimens. Acute cellular rejection and acute antibody-mediated rejection were diagnosed on the basis of the Banff criteria.

\section{Definition and Prophylaxis of Infection}

CMV infection was defined as positivity for CMV antigenemia assay $\geq 50$ cells/200,000 WBC and treated with ganciclovir. CMV antigenemia assay was performed with Light Diagnostics CMV pp65 Antigenemia kit (Chemicon International, Temecula, Calif., USA). BK virus infection was diagnosed when plasma DNA load was greater than 10,000 copies $/ \mathrm{ml}$, regardless of nephropathy. BK virus PCR was performed with Real-Q BKV quantitation kit (Biosewoom, Seoul, Korea). For PCP prophylaxis, oral trimethoprim/sulfamethoxazole $(80 / 400 \mathrm{mg})$ was administered daily for 6 months postoperatively. CMV prophylaxis was not given routinely. We checked CMV antigenemia assay periodically, and treated CMV infection when it occurred.

\section{Statistical Analysis}

All statistical analyses were performed with SPSS 18. Data are expressed as means and standard deviation. Categorical variables were compared using the $\chi^{2}$ test or Fisher's exact test, and continuous variables were compared using the t test (or Mann-Whitney U test) and ANOVA (or Kruskal-Wallis test). Mortality rates were estimated by the Kaplan-Meier method, tested by log-rank test. A two-tailed p value $<0.05$ was considered statistically significant.

\section{Results}

\section{Clinical Characteristics of the Patients}

The patients' baseline characteristics are shown in table 1 . There were more men than women in both groups. Patients' mean ages in group 1 and group 2 were $44.88 \pm 11.65$ and $42.72 \pm 10.39$ years, respectively $(\mathrm{p}=0.228)$. Etiologies of ESRD and past medical history in the two groups were not significantly different.

\section{Doses of Maintenance Immunosuppressants}

Drug levels and doses of the immunosuppressants are shown in table 2. The drug levels of tacrolimus and the doses of mPD after 6 months, 1 and 2 years after transplantation showed no difference between the two groups. MMF dose was reduced when CMV, BK virus infection and severe infection such as sepsis occurred. This decision was made by clinician's judgment. The doses of MMF (in grams/day) at 1 month, 3 months, 6 months, 1 year and 2 years postoperatively were significantly lower in group 1 than in group 2 . 
Table 1. Baseline clinical characteristics

\begin{tabular}{|c|c|c|c|}
\hline & Group $1(n=67)$ & Group $2(\mathrm{n}=87)$ & $\mathrm{p}$ value \\
\hline Sex (male/female) & $42 / 25$ & $46 / 41$ & 0.252 \\
\hline Age, years & $44.88 \pm 11.65$ & $42.72 \pm 10.39$ & 0.228 \\
\hline Dialysis (hemodialysis/peritoneal) & $57 / 6$ & $54 / 13$ & 0.139 \\
\hline Dialysis duration, month & $32.11 \pm 33.47$ & $31.04 \pm 39.09$ & 0.868 \\
\hline Etiology of ESRD & & & 0.541 \\
\hline Diabetes mellitus & 14 & 21 & \\
\hline Hypertension & 12 & 23 & \\
\hline Glomerulonephritis & 16 & 15 & \\
\hline Vesicoureteral reflux & 4 & 1 & \\
\hline Polycystic kidney disease & 5 & 5 & \\
\hline Unknown etiology & 11 & 15 & \\
\hline Other causes & 5 & 7 & \\
\hline \multicolumn{4}{|l|}{ Past medical history } \\
\hline Diabetes mellitus & 15 & 23 & 0.579 \\
\hline Hypertension & 57 & 73 & 0.843 \\
\hline Hepatitis & 6 & 2 & 0.079 \\
\hline Autoimmune disease & 0 & 1 & 1.000 \\
\hline Malignancy history & 3 & 0 & 0.080 \\
\hline Mean follow up time, months & $12.63 \pm 7.59$ & $11.10 \pm 8.16$ & 0.238 \\
\hline \multicolumn{4}{|l|}{ Donor to recipient } \\
\hline Compatible & 21 & 87 & \\
\hline $\mathrm{A} \rightarrow \mathrm{B}$ & 11 & - & \\
\hline $\mathrm{B} \rightarrow \mathrm{A}$ & 8 & - & \\
\hline $\mathrm{A} / \mathrm{B} \rightarrow \mathrm{O}$ & 18 & _- & \\
\hline $\mathrm{AB} \rightarrow \mathrm{A} / \mathrm{B} / \mathrm{O}$ & 9 & - & \\
\hline HLA mismatch $(0 / 1 / 2 / 3 / 4 / 5 / 6)$ & $4 / 0 / 7 / 20 / 7 / 18 / 11$ & $4 / 2 / 12 / 25 / 14 / 19 / 11$ & 0.790 \\
\hline HLA class I mismatch $(0 / 1 / 2 / 3 / 4)$ & $4 / 5 / 27 / 14 / 17$ & $5 / 8 / 34 / 23 / 17$ & 0.877 \\
\hline HLA class II mismatch $(0 / 1 / 2)$ & $6 / 33 / 28$ & $10 / 49 / 28$ & 0.442 \\
\hline Donor sex (male/female) & $34 / 33$ & $46 / 41$ & 0.871 \\
\hline Donor age, years & $41.88 \pm 11.65$ & $43.00 \pm 9.90$ & 0.521 \\
\hline Donor's relation with recipient & & & 0.849 \\
\hline Parents & 7 & 8 & \\
\hline Siblings & 20 & 36 & \\
\hline Children & 11 & 6 & \\
\hline Spouse & 21 & 22 & \\
\hline Others & 8 & 15 & \\
\hline
\end{tabular}

\section{Incidence of Infection}

Infections occurred in 52.2\% (35 patients) of group 1 and $40.2 \%$ (35 patients) of group 2 $(\mathrm{p}=0.138)$. However, CMV infection (16.4\% in group 1 and $5.7 \%$ in group $2, \mathrm{p}=0.031)$ and pneumonia (9.0\% in group 1 and $1.1 \%$ in group $2, \mathrm{p}=0.043$ ) occurred more often in group 1. The incidences of BK virus infection, urinary tract infection, sepsis and other infections including candida, herpes simplex virus and intra-abominal infection showed no significant difference between the two groups (table 3).

\section{Graft Rejection}

There was no hyperacute rejection or antibody-mediated rejection in either group 1 or group 2. Acute cellular rejection occurred in 3 patients (4.5\%) in group 1 and 8 patients $(9.2 \%)$ in group $2(\mathrm{p}=0.351)$. Chronic rejection occurred only in 1 patient of group 2 . There was no 
Table 2. Doses of immunosuppressants

\begin{tabular}{lccc}
\hline & Group 1 $(\mathrm{n}=67)$ & Group 2 $(\mathrm{n}=87)$ & p value \\
\hline Tacrolimus drug levels, ng/ml & & & \\
Pre KT (67/87) & $9.56 \pm 4.75$ & $11.11 \pm 6.58$ & 0.104 \\
After 1 month (65/87) & $9.17 \pm 3.22$ & $7.65 \pm 2.89$ & 0.003 \\
After 3 months (55/71) & $7.64 \pm 2.54$ & $7.83 \pm 2.88$ & 0.710 \\
After 6 months (48/56) & $7.11 \pm 2.11$ & $6.80 \pm 2.15$ & 0.463 \\
After 1 year (39/36) & $6.57 \pm 2.13$ & $5.84 \pm 2.16$ & 0.142 \\
After 2 years (4/9) & $5.48 \pm 1.81$ & $5.73 \pm 3.95$ & 0.683 \\
Mycophenolate mofetil doses, g/day & & & \\
Pre KT (67/87) & $1.51 \pm 0.29$ & $1.55 \pm 0.17$ & 0.325 \\
After 1 month (65/87) & $1.26 \pm 0.42$ & $1.40 \pm 0.39$ & 0.033 \\
After 3 months (55/71) & $1.14 \pm 0.51$ & $1.36 \pm 0.39$ & 0.011 \\
After 6 months (48/56) & $1.07 \pm 0.50$ & $1.30 \pm 0.42$ & 0.012 \\
After 1 year (39/36) & $0.88 \pm 0.52$ & $1.19 \pm 0.44$ & 0.009 \\
After 2 years (5/10) & $0.69 \pm 0.55$ & $1.25 \pm 0.49$ & 0.059 \\
Methylprednisolone doses, mg/day & & & \\
Pre KT (67/87) & $16.84 \pm 6.01$ & $15.91 \pm 0.86$ & 0.214 \\
After 1 month (65/87) & $11.97 \pm 2.31$ & $11.59 \pm 2.70$ & 0.358 \\
After 3 months (55/71) & $9.09 \pm 1.48$ & $8.31 \pm 2.13$ & 0.022 \\
After 6 months (48/56) & $7.33 \pm 1.39$ & $7.50 \pm 3.46$ & 0.755 \\
After 1 year (39/36) & $5.33 \pm 1.80$ & $6.39 \pm 10.00$ & 0.519 \\
After 2 years (4/10) & $4.00 \pm 0.00$ & $3.40 \pm 1.65$ & 0.496 \\
\hline
\end{tabular}

Table 3. Incidence of infection

\begin{tabular}{lccl}
\hline & Group 1 $(\mathrm{n}=67)$ & Group 2 $(\mathrm{n}=87)$ & p value \\
\hline Incidence of infection & $35(52.2 \%)$ & $35(40.2 \%)$ & 0.138 \\
Cytomegalovirus & $11(16.4 \%)$ & $5(5.7 \%)$ & 0.031 \\
BK virus & $9(13.4 \%)$ & $7(8.0 \%)$ & 0.277 \\
Urinary tract infection & $6(9.0 \%)$ & $14(16.1 \%)$ & 0.192 \\
Pneumonia & $6(9.0 \%)$ & $1(1.1 \%)$ & 0.043 \\
Sepsis & $4(6.0 \%)$ & $2(2.3 \%)$ & 0.404 \\
Others & $22(32.8 \%)$ & $16(18.4 \%)$ & 0.039 \\
\hline
\end{tabular}

significant difference in the incidence of acute cellular rejection and chronic rejection between the two groups (table 4).

\section{Serum Creatinine Level}

After renal transplantation, creatinine levels were not significantly different in the two groups. Preoperative levels of creatinine (in $\mu \mathrm{mol} / \mathrm{l}$ ) in group 1 and group 2 were $752.28 \pm$ 257.24 and $770.85 \pm 316.47$, respectively $(\mathrm{p}=0.692)$. Postoperative values of creatinine were $86.63 \pm 18.56$ vs. $97.24 \pm 27.4$ at 2 years $(\mathrm{p}=0.614)($ table 5$)$.

\section{Incidence of Malignancy}

Malignancy occurred in two patients of group 1 and none in group 2 (table 6). One patient developed skin squamous cell carcinoma on the anterior chest wall 8 months after renal 
Table 4. Graft rejection

\begin{tabular}{llll}
\hline & Group 1 $(\mathrm{n}=67)$ & Group 2 $(\mathrm{n}=87)$ & p value \\
\hline Hyperacute rejection & $0(0 \%)$ & $0(0 \%)$ & - \\
Acute cellular rejection & $3(4.5 \%)$ & $8(9.2 \%)$ & 0.351 \\
Antibody-mediated rejection & $0(0 \%)$ & $0(0 \%)$ & - \\
Chronic rejection & $0(0 \%)$ & $1(1.1 \%)$ & 1.000 \\
\hline
\end{tabular}

Table 5. Serum creatinine levels $(\mu \mathrm{mol} / \mathrm{l})$

\begin{tabular}{lccc}
\hline & Group 1 $(\mathrm{n}=67)$ & Group 2 $(\mathrm{n}=87)$ & p value \\
\hline Pre-transplantation (67/87) & $752.28 \pm 257.24$ & $770.85 \pm 316.47$ & 0.692 \\
After 1 month (66/87) & $106.96 \pm 118.46$ & $91.05 \pm 29.17$ & 0.223 \\
After 3 months (55/71) & $103.43 \pm 25.64$ & $99.01 \pm 26.52$ & 0.326 \\
After 6 months (48/56) & $106.96 \pm 27.4$ & $99.01 \pm 28.29$ & 0.144 \\
After 1 year (39/36) & $102.54 \pm 28.29$ & $93.7 \pm 26.52$ & 0.152 \\
After 2 years (4/9) & $86.63 \pm 18.56$ & $97.24 \pm 27.4$ & 0.614 \\
\hline
\end{tabular}

Table 6. Incidence of malignancy

\begin{tabular}{llll}
\hline & Group 1 $(\mathrm{n}=67)$ & Group 2 $(\mathrm{n}=87)$ & $\mathrm{p}$ value \\
\hline Malignancy & $2(3.0 \%)$ & $0(0 \%)$ & 0.188 \\
\hline
\end{tabular}

Table 7. Mortality

\begin{tabular}{llll}
\hline & Group 1 $(\mathrm{n}=67)$ & Group 2 $(\mathrm{n}=87)$ & $\mathrm{p}$ value \\
\hline Mortality & $3(4.5 \%)$ & $0(0 \%)$ & 0.053 \\
Graft failure & $1(1.5 \%)$ & $0(0 \%)$ & 0.435 \\
\hline
\end{tabular}

transplantation. This was removed by excisional biopsy. The other patient was diagnosed with parathyroid cancer at 5 months after transplantation. In this patient, calcium level increased gradually after renal transplantation. After ultrasound and parathyroid scan, parathyroid adenoma was suspected. However, parathyroid carcinoma was diagnosed after parathyroidectomy.

\section{Mortality}

Three patients (4.5\%) died in group 1, whereas there were no deaths in group 2 (table 7). One patient died from septic shock associated with urinary sepsis. Fungal infective endocarditis (Aspergillus) was uncontrollable in another patient in spite of mitral valve replacement operation. The third patient was improving from pneumonia, but died from metabolic acidosis of unknown origin. Therefore, 2 of 3 deaths were due to infection. Graft survival was $98.5 \%$ in group 1 and $100 \%$ in group 2 ( $p=0.435)$. One patient experienced graft failure. However, unexpected vessel kinking after operation was the cause of graft failure. Therefore, no graft failure occurred by graft rejection. 


\section{Discussion}

In the incompatible $\mathrm{KT}$, hyperacute rejection occurs due to preformed $\mathrm{ABO}$ antibody or HLA antibody. Plasmapheresis removes the preformed antibody. Rituximab (an anti-CD20 antibody), which effectively depletes B lymphocytes, has a role in preventing new antibody formation until accommodation occurs. Thus, the present immunosuppressive protocol in incompatible KT consists of (1) pre-transplant desensitization with plasmapheresis, rituximab and anti-CD25 monoclonal antibody, and (2) the standard maintenance immunosuppression of MMF, tacrolimus and steroid at similar doses used in ABO-compatible KT. Because the additional use of rituximab may result in more immunosuppression, the initial standard doses of maintenance immunosuppressants could result in over-immunosuppression. However, the optimal doses of maintenance immunosuppressants in these patients are not known. The occurrence of serious infections is one of the important problems associated with over-immunosuppression. There have been several studies that evaluated the association of rituximab use with infectious complications. In a study of highly sensitized renal transplant recipients [15], 38 patients were treated with rituximab and 26 patients received comparable treatment but without rituximab. Severe infectious episodes were not statistically different between groups and it was concluded that rituximab therapy was not associated with an increased risk of severe infection. In contrast, several other studies suggested the increased risk of infectious complications. In a study of 77 kidney transplant patients who received rituximab therapy for various reasons and 902 control patients who received no rituximab [16], the incidence of infection was 45.45 and 53.9\%, respectively, and showed no difference. However, the mortality related to an infectious disease was significantly higher in the rituximab group ( 9.09 vs. $1.55 \%$ ). Recently, an increased risk of infectious complications was also reported in ABO-IKT [17]. In this study, 21 consecutive recipients who underwent $\mathrm{ABO}-\mathrm{IKT}$ were compared with ABO-compatible KT recipients. During the follow-up for $15.7 \pm 8.3$ months, the rate of viral infections including CMV, herpes simplex virus, varicella zoster virus and polyoma virus was significantly increased in ABO-IKT ( 50 vs. $21 \%$, $\mathrm{p}=0.038$ ) despite comparable tacrolimus trough levels and MMF and steroid doses. In the present study, the overall incidence of infection in the rituximab-treated group was not different from the control group. However, CMV infection and pneumonia occurred more frequently in the rituximab-treated group. In addition, infection was the cause of death in 2 patients of total 3 deaths in the rituximab-treated group. These findings suggest the need to reduce maintenance immunosuppression in rituximab-treated $\mathrm{KT}$.

In our study, we performed plasmapheresis for desensitization and did not give the patients IVIG. Most studies of ABO-IKT gave the patients IVIG. There are a few studies in which administration of IVIG was not performed in all patients $[18,19]$. In these studies, no serious infection occurred. Although plasmapheresis does not seem to be associated with serious infections, the possible role in the increased risk of infection cannot be excluded.

The main concern associated with reduction in immunosuppression is the occurrence of rejection. There have been trials to reduce the rituximab dose from 500 to $200 \mathrm{mg} / \mathrm{body}$ to prevent major infectious complications [13,20]. Shirakawa et al. [13] compared the results of a low dose of rituximab ( $200 \mathrm{mg} /$ body) to a usual dose of rituximab (500 mg/body). The group treated with the low dose of rituximab showed excellent comparable results. So far, however, there has been no data regarding the reduction of maintenance immunosuppressants in rituximab-treated $\mathrm{KT}$. In this study, we reduced MMF when infection occurred. The doses of MMF (in g/day) until 2 years postoperatively were lower in group 1 than in group 2 , while kidney function was not different in the two groups. In addition, the incidence of acute cellular rejection and chronic rejection was not higher in the rituximab-treated group (group 1) who were maintained on lower doses of MMF. One graft was lost in the rituximab- 
treated group, but anatomical vessel kinking was the cause of graft failure. Though the optimum level of dose reduction was not determined in this study, the findings suggest that the dose of MMF could be reduced without increasing the risk of rejection in these patients.

Our study has several limitations. This study was a retrospective cohort study. Thus, MMF dose was reduced in incompatible KT not from the early postoperative period, but only after the occurrence of serious infection. Second, the follow-up duration was short because our institute started ABO-IKT in 2009. Third, we did not analyze the data at each dose of rituximab because of the small sample size. Lastly, the possible role of plasmapheresis in the increased risk of infection cannot be excluded.

In conclusion, serious infectious complication was increased in rituximab-treated KT and our study suggests the plausibility of reducing maintenance immunosuppressants in these patients. It might be adequate to reduce MMF dose from the early postoperative period. Prospective randomized controlled studies are warranted to determine the optimal dose of maintenance immunosuppression in these patients.

\section{Disclosure Statement}

There are no conflicts of interest.

\section{References}

1 Alexandre GP, Squifflet JP, De Bruyere M, Latinne D, Reding R, Gianello P, Carlier M, Pirson Y: Present experiences in a series of $26 \mathrm{ABO}$-incompatible living donor renal allografts. Transplant Proc 1987; 19:4538-4542.

-2 Takahashi K: Recent findings in abo-incompatible kidney transplantation: Classification and therapeutic strategy for acute antibody-mediated rejection due to ABO-blood-group-related antigens during the critical period preceding the establishment of accommodation. Clin Exp Nephrol 2007;11: $128-141$.

-3 Sawada T, Fuchinoue S, Kawase T, Kubota K, Teraoka S: Preconditioning regimen consisting of antiCD20 monoclonal antibody infusions, splenectomy and DFPP-enabled non-responders to undergo abo-incompatible kidney transplantation. Clin Transplant 2004;18:254-260.

-4 Sawada T, Fuchinoue S, Teraoka S: Successful A1-to-O ABO-incompatible kidney transplantation after a preconditioning regimen consisting of anti-CD20 monoclonal antibody infusions, splenectomy, and double-filtration plasmapheresis. Transplantation 2002;74:1207-1210.

-5 Tyden G, Kumlien G, Fehrman I: Successful ABO-incompatible kidney transplantations without splenectomy using antigen-specific immunoadsorption and rituximab. Transplantation 2003;76: $730-731$.

-6 Sonnenday CJ, Warren DS, Cooper M, Samaniego M, Haas M, King KE, Shirey RS, Simpkins CE, Montgomery RA: Plasmapheresis, CMV hyperimmune globulin, and anti-CD20 allow ABO-incompatible renal transplantation without splenectomy. Am J Transplant 2004;4:1315-1322.

-7 Fuchinoue S, Ishii Y, Sawada T, Murakami T, Iwadoh K, Sannomiya A, Koyama I, Kubota K, Tojimbara T, Nakajima I, Teraoka S: The 5-year outcome of ABO-incompatible kidney transplantation with rituximab induction. Transplantation 2011;91:853-857.

-8 Genberg H, Kumlien G, Wennberg L, Berg U, Tyden G: ABO-incompatible kidney transplantation using antigen-specific immunoadsorption and rituximab: a 3-year follow-up. Transplantation 2008; $85: 1745-1754$.

-9 Montgomery RA, Lonze BE, King KE, Kraus ES, Kucirka LM, Locke JE, Warren DS, Simpkins CE, Dagher NN, Singer AL, Zachary AA, Segev DL: Desensitization in HLA-incompatible kidney recipients and survival. N Engl J Med 2011;365:318-326.

-10 Linares L, Cofan F, Cervera C, Ricart MJ, Oppenheimer F, Campistol JM, Moreno A: Infection-related mortality in a large cohort of renal transplant recipients. Transplant Proc 2007;39:2225-2227. 
11 Budde K, Becker T, Arns W, Sommerer C, Reinke P, Eisenberger U, Kramer S, Fischer W, Gschaidmeier H, Pietruck F: Everolimus-based, calcineurin-inhibitor-free regimen in recipients of de-novo kidney transplants: an open-label, randomised, controlled trial. Lancet 2011;377:837-847.

- 12 Helal I, Chan L: Steroid and calcineurin inhibitor-sparing protocols in kidney transplantation. Transplant Proc 2011;43:472-477.

-13 Shirakawa H, Ishida H, Shimizu T, Omoto K, Iida S, Toki D, Tanabe K: The low dose of rituximab in ABO-incompatible kidney transplantation without a splenectomy: a single-center experience. Clin Transplant 2011;25:878-884.

14 Takagi T, Ishida H, Shirakawa H, Shimizu T, Tanabe K: Evaluation of low-dose rituximab induction therapy in living related kidney transplantation. Transplantation 2010;89:1466-1470.

-15 Scemla A, Loupy A, Candon S, Mamzer MF, Martinez F, Zuber J, Sberro R, Legendre C, Thervet E: Incidence of infectious complications in highly sensitized renal transplant recipients treated by rituximab: a case-controlled study. Transplantation 2010;90:1180-1184.

-16 Kamar N, Milioto O, Puissant-Lubrano B, Esposito L, Pierre MC, Mohamed AO, Lavayssiere L, Cointault O, Ribes D, Cardeau I, Nogier MB, Durand D, Abbal M, Blancher A, Rostaing L: Incidence and predictive factors for infectious disease after rituximab therapy in kidney-transplant patients. Am J Transplant 2010;10:89-98.

-17 Habicht A, Broker V, Blume C, Lorenzen J, Schiffer M, Richter N, Klempnauer J, Haller H, Lehner F, Schwarz A: Increase of infectious complications in ABO-incompatible kidney transplant recipients - a single centre experience. Nephrol Dial Transplant 2011;26:4124-4131.

-18 Tanabe K, Ishida H, Shimizu T, Omoto K, Shirakawa H, Tokumoto T: Evaluation of two different preconditioning regimens for $\mathrm{ABO}$-incompatible living kidney donor transplantation. A comparison of splenectomy vs. rituximab-treated non-splenectomy preconditioning regimens. Contrib Nephrol 2009;162:61-74.

-19 Uchida J, Machida Y, Iwai T, Naganuma T, Kitamoto K, Iguchi T, Maeda S, Kamada Y, Kuwabara N, Kim T, Nakatani T: Desensitization protocol in highly HLA-sensitized and ABO-incompatible high titer kidney transplantation. Transplant Proc 2010;42:3998-4002.

-20 Chikaraishi T, Sasaki H, Tsutsumi H, Miyano S, Nakazawa R, Nakano T, Kitajima K, Kudo H, Takahashi T, Sato Y, Kimura K: ABO blood type incompatible kidney transplantation without splenectomy prepared with plasma exchange and rituximab. Transplant Proc 2008;40:3445-3447. 